\title{
AOR
}

Selected Papers of \#AoIR2021:

The 22nd Annual Conference of the

Association of Internet Researchers

Virtual Event / 13-16 Oct 2021

\section{CART NARCS AND THE ENGINEERING OF SOCIAL SHAMING AS ENTERTAINMENT}

CJ Reynolds

The Hebrew University of Jerusalem

Nicholas John

The Hebrew University of Jerusalem

The typical Cart Narcs YouTube video opens with "Agent Sebastian" walking through a grocery store parking lot. Sebastian briefly explains the video's purpose - "We shame people who don't put their carts back" - as he looks for a cart miscreant to confront. When he spots a target, he runs towards them making siren noises, outfitted in a Cart Narcs t-shirt and a police-style utility vest. "Cart Narcs!" he shouts. "That's not where the cart goes!" From here, the videos take one of two routes. In a minority of cases, the target returns the cart. In most cases, though, the interaction escalates and the target ends up yelling and swearing at Sebastian, who calmly insists that he is just promoting considerate behavior.

With around 160 videos, 160,000 subscribers, and 18 million views, Cart Narcs is an "elite" YouTube channel (Rieder et al., 2020). Cart Narcs was first launched in August 2018 under the auspices of The Woody Show, an irreverent shock jock radio show. Since February 2020, the Cart Narcs channel has been a stand-alone enterprise, far outstripping its original home in popularity. In addition to the YouTube channel, Cart Narcs also has a Facebook page (413,000 followers) and Instagram account $(355,000$ followers) that posts Stories sent in by a community of fans, or "Narcateers," purportedly dedicated to "promoting personal responsibility" (Cart Narcs, 2021) by shaming people into returning their shopping cart.

At first glance, Cart Narcs videos resemble established entertainment featuring real people (e.g. Candid Camera). Such shows construct a situation that resembles everyday life but is actually carefully engineered, and rely for their humor on the gap in knowledge between presenter and audience on the one hand, and the unknowing sap on the other. Cart Narcs videos have a number of memetic qualities (Shifman, 2012) that resonate with prank videos (Hobbes and Grafe, 2015), where humor is derived from the confusion and anger of the prank subject, as well as with shock jock radio and 
"public freakout" videos. Despite these similarities, however, we identify significant features in Cart Narcs videos that distinguish them: the lack of a debrief; the positioning of the Cart Narc as the "nice guy" in the interaction; and the Cart Narc's claim to the moral high ground. These features each remove a redemptive moment present in analogous types of content, resulting in a communicative interaction engineered to anger people.

We ask how Agent Sebastian produces such a powerful emotional response to his seemingly innocent request that people return their shopping cart, and what his strategies are for eliciting anger. We then ask why he does this, and what the logic of this form of media content might signify.

We selected the 15 most viewed videos as our sample. These videos range from 235,000 to 1.2 million YouTube views. We conducted a close reading of these videos, drawing also on related paratexts, including interviews with Agent Sebastian, viewersubmitted Instagram Stories, and the fan-run subreddit. We carefully noted the patterns of interaction in these videos, including how the Cart Narc framed his motivations for viewers and the people he confronted, what actions were likely to escalate conflict, and, by contrast, the ways that people tried to end, intervene in, or de-escalate such confrontations.

Preliminary analysis suggests that the success of Cart Narcs lies in the creation of deliberately discombobulating communicative situations. The videos can thus be read as breaching experiments that operate through the mechanism of callout culture. Garfinkel (1967) explained that breaching experiments "start with familiar scenes and ask what can be done to make trouble" (37), which the Cart Narc does by calling people out over a situation that does not warrant confrontation with the goal of generating "the socially structured affects of anxiety, shame, guilt, and indignation" (38). In terms of how he does this, we noted repeated strategies including calling people "lazybones," repeatedly calling himself a "nice guy" when criticized, placing magnetic bumper stickers on people's cars (which irritates them deeply), blocking their cars with carts, and intransigently refusing to end conversations until people admit fault and return their cart. The cumulative effect of these strategies creates escalating irritation in people reacting to his multiple social breaches: the saps do not understand why they are being called out - and filmed - for not returning their shopping cart, and they do not appreciate being engaged in debate when they have asked Agent Sebastian to leave them alone.

These strategies point towards three unique features: the lack of a debrief, Agent Sebastian calling himself a "nice guy," and his claim to the moral high ground. Unlike prank videos where the prankster breaks character to explain the joke ("Smile! You're on Candid Camera!"), Cart Narcs participants are denied this catharsis. Debriefing puts the prankster and the sap on equal footing and restores social solidarity. Without this, the sap is left with unresolved anger and confusion.

Secondly, in prank content, it is the prankster who is rude or unreasonable. However, by positioning himself as the "nice guy," Agent Sebastian flips this, and implicitly frames the sap as reprehensible. Thus, he engineers interactions that make the anger directed at him appear unreasonable. While further infuriating the saps, it also creates plausible 
deniability, both for himself and the audience that in fact the whole point of the interaction is to make the saps angry.

Finally, by engineering a communication situation around returning shopping carts, the Cart Narc is able to take the moral high ground and leaves saps few options: concede and return the cart; tell the Cart Narc to mind his own business; ignore him; or - and this is the reaction the situation is designed to provide - argue with him about why their behavior is normative, justifiable, or simply not a big deal.

The Cart Narcs videos instantiate the logic of a communication situation that has been engineered with the sole intent of generating social media engagement. Agent Sebastian deliberately pushes his targets to an emotional breaking point without ever explaining to them why. The plausible deniability created by the combination of his "nice guy" posturing and his appropriation of the moral high ground allows the audience to enjoy these videos for their real purpose - watching people freak out in public - without having to admit to themselves the true source of their pleasure. Cart Narcs videos are a revealing case study in how the economy-driven logic of participatory culture (Shifman 2012) produces undesirable types of content when it overwhelms or wholly replaces social and aesthetic logics. Borrowing from pre-existing genres - minus crucial redemptive aspects - Cart Narcs videos are a hybrid concoction that trade people's anger for monetized views, cloaked by the pretense of a social mission.

\section{References}

Cart Narcs. "About." Cart Narcs YouTube Channel. Accessed 14 April 2021. Available at: https://www.youtube.com/channel/UCIMUlr8yHymYgSe58DpUH7w

Garfinkel, Harold. 1967. Studies in Ethnomethodology. Englewood Cliffs, New Jersey: Prentice-Hall.

Hobbs, Renee, and Silke Grafe. 2015. "YouTube Pranking across Cultures." First Monday, June. https://doi.org/10.5210/fm.v20i7.5981.

Rieder, Bernhard, Òscar Coromina, and Ariadna Matamoros-Fernández. 2020. "Mapping YouTube:" First Monday, July. https://doi.org/10.5210/fm.v25i8.10667.

Shifman, Limor. 2012. "An Anatomy of a YouTube Meme.” New Media \& Society 14 (2): 187-203. https://doi.org/10.1177/1461444811412160. 